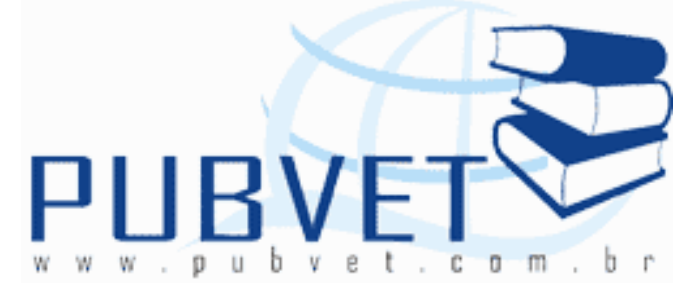

PUBVET, Publicações em Medicina Veterinária e Zootecnia.

\title{
Análise sensorial discriminativa de méis de laranjeira puro e adicionados com xarope de glicose
}

Luciana Godoy Viana ${ }^{1}$; Juliana Guimarães Fonseca ${ }^{1}$; Letícia Mariano da Costa ${ }^{1}$;

Daniel Bedo Assupção Castro; ${ }^{1}$ Maísa Curtolo e Marta Regina Verruma

Bernardi ${ }^{2}$

${ }^{1}$ Graduandos da Universidade Federal de São Carlos - Campus Araras-SP, Rodovia Anhanguera, Km 174 Araras (SP),

e-mail: luciana.godoyv@hotmail.com;

${ }^{2}$ Profa da Universidade Federal de São Carlos, DTAiSER - Departamento de Tecnologia Agroindustrial e Sócio-Economia Rural.

\section{Resumo}

O objetivo deste trabalho foi avaliar se provadores não treinados conseguem verificar diferença sensorial entre mel de laranjeira puro, mel com 20\% de xarope de glicose e mel com $50 \%$ de xarope de glicose. Para tal foi utilizado o teste discriminativo de ordenação com 40 provadores não treinados. Os atributos avaliados foram: cor (claro-escuro), aroma (menos característicomais característico), consistência (menos consistente-mais consistente), sabor doce (menos doce-mais doce) e preferência (menos gostou-mais gostou). Verificou-se que provadores identificaram diferença entre o mel puro das amostras de mel adicionadas de xarope de glicose apenas para o atributo consistência; a amostra de mel com $50 \%$ de xarope de glicose apresentou 
menor consistência possivelmente pela composição do xarope de glicose possuir maior teor de água. Sendo assim, a consistência poder ser considerada uma característica para distinção do mel puro.

Palavras-chave: mel, análise sensorial, consistência, atributos sensoriais, glicose de milho.

\title{
Sensory analysis discriminative of pure honey from citrus flowers and honey with glucose syrup added
}

\begin{abstract}
This paper aims to evaluate if the tasters not trained can check sensory differences and preferences in relation to pure honey from citrus flowers, honey with $20 \%$ of glucose syrup and honey with $50 \%$ of glucose syrup. For this study was used the preference ordination test with 40 tasters not trained. The evaluated attributes were: color (clear-dark), smell (less characteristicmore characteristic), consistency (less consistent-more consistent), sweet flavor (less sweet-more sweet) and preference (less liked- more liked). It was found that the tasters identified difference between pure honey of the modified sample with added glucose syrup only for consistency attribute; the sample honey with $50 \%$ of glucose syrup presented the lowest consistency probably because of the composition of de glucose syrup has more water. Consistency may be considered a characteristic to distinction of pure honey.
\end{abstract}

Keywords: honey, sensory analysis, consistency, sensory attributes, corn glucose.

\section{INTRODUÇÃO}

O mel é produzido através da relação simbiótica entre plantas e abelhas (CAMPOS, 2003; MARCHINI et al., 2005), sendo o mais importante produto apícola. O Brasil exporta cerca de 1,74 mil toneladas de mel principalmente para os Estados Unidos, Alemanha e Reino Unido, apresentando uma receita até junho de 2011 de US\$ 5,63 milhões (SEBRAE, 2012). 
Em sua composição há majoritariamente frutose, glicose e água, também apresenta teores de proteínas, vitaminas, sais minerais, entre outros (VENTURINI et al., 2007).

Atualmente, existem parâmetros que visam estabelecer a qualidade e controle do mel. De acordo com a instrução normativa $n^{\circ} 11$ de 2000, que regulamenta a identidade e qualidade do mel, as características sensoriais são: consistência, cor, aroma e sabor (MAPA, 2000). A consistência do mel depende grandemente do seu conteúdo de água e está assim ligada a sua densidade relativa, já a coloração, aroma e sabor do mel variam de acordo com a sua origem floral (MAPA, 2000); (PEREIRA et al., 2003).

No Brasil, há porcentagens variáveis de adulteração de mel (ARAUCO et al., 2008) sendo que o principal método de adulteração é a adição de xarope de glicose (WHITE \& DONER, 1978); (MORALES et al., 2008), porém, há poucos estudos que a relacione a qualidade este fato com a análise sensorial. Provadores em um teste pareado avaliaram a capacidade de discernir o mel de jandaíra (Melipona subnitida) de amostras com a adição de $20 \%$ de mel de apis e outra contendo $20 \%$ de xarope de glicose, identificando as amostras quanto ao aroma, sabor e viscosidade. Como o mel de jandaíra é considerado com aroma mais forte, menos viscoso e menos doce do que o mel de apis, os provadores tiveram facilidade em identificá-lo. A não familiaridade dos provadores com o mel de jandaíra pode ter influenciado no grau de aceitação da amostra de mel puro (MESQUITA et al., 2007).

A análise sensorial corresponde à avaliação das características sensoriais dos produtos através dos sentidos humanos (PIANA et al., 2004). Quando se deseja encontrar diferenças sensoriais entre produtos a respeito de seus atributos, o teste discriminativo de ordenação pode ser utilizado.

Desta maneira, este trabalho tem como objetivo avaliar se os provadores conseguem verificar se há diferenças sensoriais e a preferência, em relação ao mel puro, mel com $20 \%$ de xarope de glicose e mel com $50 \%$ de xarope de glicose. 


\section{MATERIAL E MÉTODOS}

O mel de florada de laranjeira foi adquirido no município de Araras, inspecionado pelo SIM (Serviço de Inspeção Municipal), Secretaria Municipal de Serviços Urbanos e Rurais (SMSUR), atua dentro das especificações exigidas pela resolução no12 de 1978 da Comissão Nacional de Normas e Padrões para Alimentos e armazenado no laboratório de análise sensorial do Campus Araras/UFSCar em temperatura de aproximadamente $25^{\circ} \mathrm{C}$.

O mel puro foi divido em três grupos sendo um grupo composto por mel puro e duas amostras modificadas com $20 \%(\mathrm{~m} / \mathrm{m})$ e $50 \%(\mathrm{~m} / \mathrm{m})$ de xarope de glicose.

A análise sensorial foi realizada no laboratório de análise sensorial do Campus Araras/UFSCar, com quarenta provadores voluntários não treinados. 0 teste discriminativo de ordenação (ABNT, 1994) foi aplicado para os atributos de cor, aroma, sabor e consistência e quanto à preferência. Cada provador recebeu um copo de água para lavar o palato entre as amostras e cerca de $20 \mathrm{~g}$ de cada amostra, servidas a temperatura ambiente em copos descartáveis de $50 \mathrm{~mL}$ com colheres de plástico. As amostras foram codificadas com três letras aleatórias: sendo uma amostra referente ao mel puro, outra ao mel com $20 \%$ de xarope de glicose e uma amostra com 50\% de xarope de glicose.

A análise estatística dos dados foi feita através do teste de Friedman (ABNT, 1994), que define se as amostras diferem ou não significativamente ao nível de $5 \%$ de significância. Uma análise discriminante foi aplicada nas ordens sugeridas pelos provadores em grupos definidos a priori na tentativa de visualizar graficamente a discriminação nos atributos sensoriais e nas amostras de mel puro e modificadas.

\section{RESULTADOS E DISCUSSÃO}

Os resultados obtidos do teste de ordenação, onde as três amostras foram avaliadas para os atributos cor, consistência, aroma, sabor e preferência, estão apresentados na Tabela 1 . Sendo que apenas o atributo consistência apresentou diferença significativa entre as amostras. 
A coloração do mel de laranjeira é classificada como âmbar, possuindo menor intensidade de cor quando comparado a outros sete méis oriundos de diferentes floradas (SERRA, s.d). Em um estudo realizado por Modesta (2007), $52,5 \%$ dos consumidores de mel avaliam a cor ao adquirir o produto, sendo que geralmente preferem o mel de coloração mais clara. Porém, no presente estudo os provadores não foram capazes de identificar diferenças entre a coloração do mel puro e as amostras com a adição de glicose (Tabela 1).

Tabela 1. Somatório das notas atribuídas pelos provadores aos diferentes atributos.

\begin{tabular}{lccccc}
\hline Amostras & Cor & Consistência & Aroma & Sabor & Preferência \\
\hline Mel puro & $71^{\mathrm{a}}$ & $99^{\mathrm{a}}$ & $83^{\mathrm{a}}$ & $72^{\mathrm{a}}$ & $83^{\mathrm{a}}$ \\
$\mathbf{2 0 \%}$ xarope de glicose & $85^{\mathrm{a}}$ & $83^{\mathrm{a}}$ & $88^{\mathrm{a}}$ & $88^{\mathrm{a}}$ & $84^{\mathrm{a}}$ \\
$\mathbf{5 0 \%}$ xarope de glicose & $78^{\mathrm{a}}$ & $58^{\mathrm{b}}$ & $69^{\mathrm{a}}$ & $80^{\mathrm{a}}$ & $73^{\mathrm{a}}$
\end{tabular}

Teste de Friedman a $5 \%$ de significância - valores seguidos por letras iguais não diferem estatisticamente. Diferença mínima significativa $=21$

Bastos et al. (2002), ao analisar sensorialmente méis de eucalipto e laranjeira através da Análise Descritiva Quantitativa demonstraram que o aroma e sabor atribuídos ao mel estão associados a compostos voláteis. Para o mel de laranjeira, o benzaldeído foi caracterizado como adocicado e laranja, este composto é considerado um importante fator na determinação destas duas características sensoriais.

A consistência do mel está relacionada com a quantidade de água (PEREIRA et al., 2003), quanto menor o conteúdo de água, maior será a consistência e densidade. Como o teor de umidade no mel de laranjeira é de $19,16 \% \pm 0,57$ (SALGADO et al., 2008) e o teor de água no xarope de glicose é de 20\% (GALLI et al., 1996), é possível inferir que ao se utilizar uma maior concentração de xarope de glicose, aumenta-se o teor de água e consequentemente há redução da consistência. Desta forma, a consistência foi 
o único atributo que apresentou diferença significativa (Tabela 1) entre as amostras, sendo que a amostra com $50 \%$ de xarope de glicose foi a que apresentou menor consistência (Tabela 1).

A análise discriminante proporcionou uma explicação de $82,99 \%$ para o primeiro fator e $17,01 \%$ para o segundo fator, o que explica $100 \%$ da variância do experimento, permitindo visualizar nas variáveis (Figura 1) a significância encontrada no teste de Friedman (Tabela 1) para consistência, o que a destaca como característica para distinguir o mel puro, além de apontar a característica aroma como outra variável discriminante.

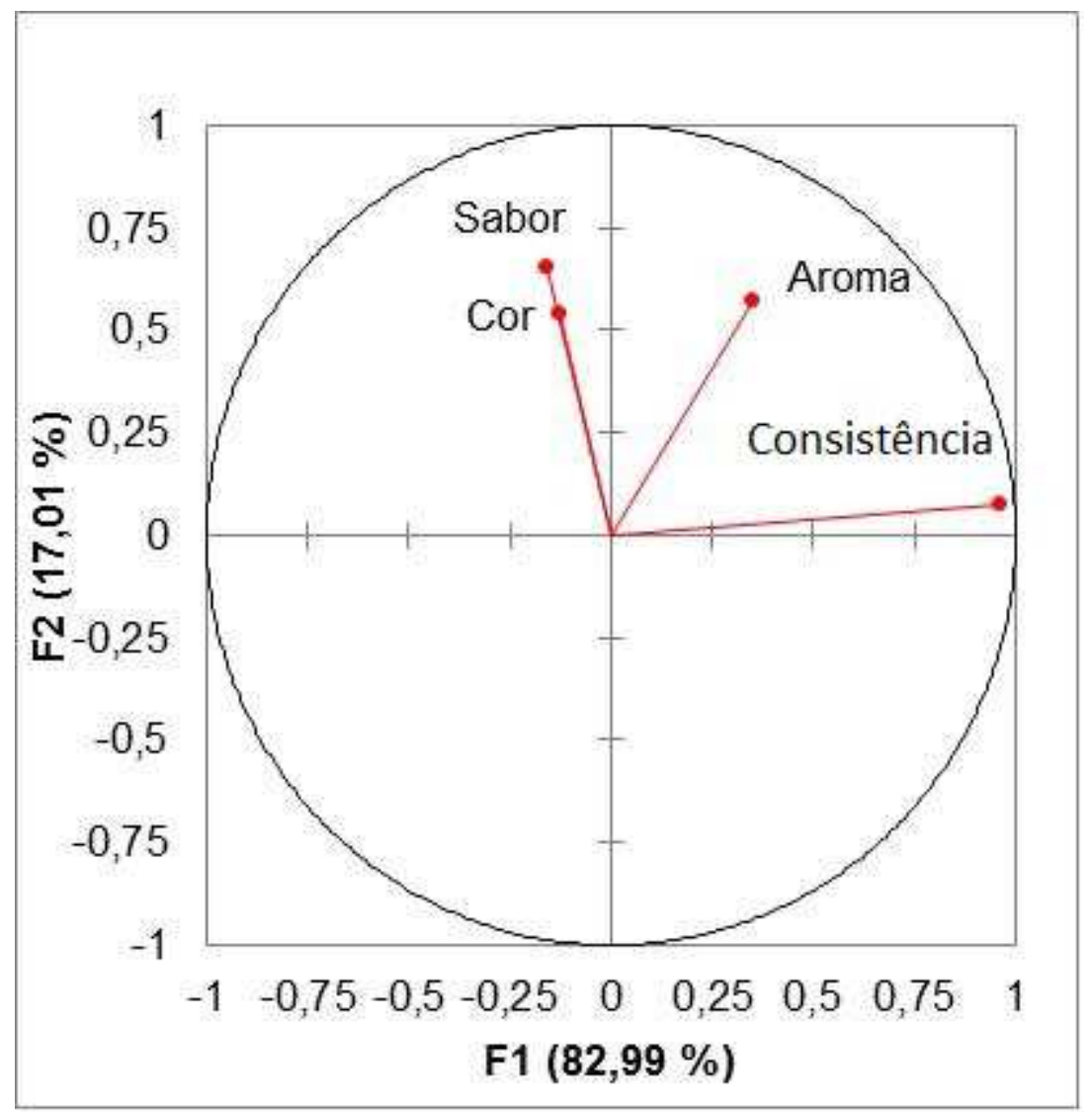

Figura 1. Localização dos atributos cor, consistência, aroma e sabor no espaço do primeiro e segundo fator.

A tentativa de discriminar as observações (Figura 2) feitas pelos provadores não proporcionou clusters, provocando erros de interpretação ao 
aparecer observações sobrepostas. Todavia o gráfico posicionou as amostras A e C mais distantes entre si, enquanto a $\mathrm{B}$ mais dispersa no gráfico (Figura 2).

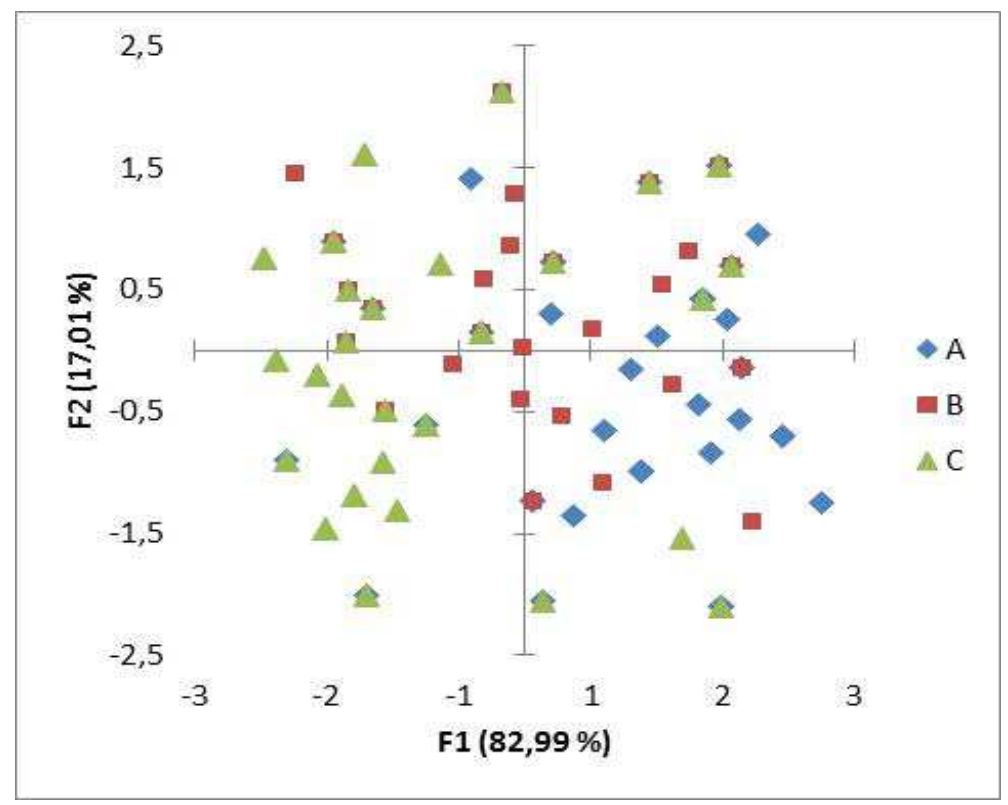

Figura 2. Disposição das observações das amostras de mel puro(A), mel com $20 \%$ de xarope de glicose (B) e mel com 50\% de xarope de glicose(C) no espaço do primeiro e segundo fator.

A não formação de clusters e a maior dispersão da amostra B podem ser melhores explicadas através uma matriz de confusão ou de erros com os grupos definidos a priori (Tabela 2). Para as amostras de mel puro (A) e o modificado em 50\% (C), nota-se uma maior porcentagem de acerto, correspondendo $70 \%$ e $72,50 \%$ respectivamente, sugerindo uma melhor percepção da modificação e as colocando mais distantes entre si (Figura 2). Porém para a amostra com $20 \%$ de xarope de glicose (B), a porcentagem de acerto foi de $52,50 \%$, sendo mais confundida com o mel puro (A) $(27,50 \%)$, o que mostra a menor capacidade do provador para diferenciar mel puro de um mel pouco modificado. 
Tabela 2. Matriz de erros dos grupos definidos a priori.

\begin{tabular}{llllll}
\hline & Mel puro & $\mathbf{2 0 \%}$ & $\mathbf{5 0 \%}$ & Total & \% correto \\
\hline Mel puro & 28 & 7 & 5 & 40 & $70,00 \%$ \\
$\mathbf{2 0 \%}$ & 11 & 21 & 8 & 40 & $52,50 \%$ \\
$\mathbf{5 0 \%}$ & 6 & 5 & 2 & 40 & $72,50 \%$
\end{tabular}

Legenda: a denominação $20 \%$ é referente à amostra de mel com $20 \%$ de xarope de glicose, e $50 \%$ refere-se à amostra de mel com $50 \%$ de xarope de glicose.

A preferência por determinado produto é de grande importância, pois representa o conjunto de todas as percepções sensoriais bem como o julgamento do consumidor sobre o produto, porém, não houve diferença significativa entre as amostras para este atributo (Tabela 1), mostrando que os provadores não preferiram uma amostra, apesar da menor média obtida para a amostra com $50 \%$ de xarope de glicose (C).

\section{CONCLUSÃO}

A consistência poder ser considerada uma característica diferenciar o mel puro das outras amostras adicionadas de glicose. Como os provadores não são capazes de identificar sensorialmente diferenças entre o mel com menores teores de xarope de glicose $(20 \%)$, cabe aos órgãos fiscalizadores realizar a avaliação do produto por outros testes mais específicos, como a análise físicoquímica.

\section{REFERÊNCIAS BIBLIOGRÁFICAS}

ABNT. ASSOCIAÇÃO BRASILEIRA DE NORMAS TÉCNICAS. Teste de ordenação em análise sensorial. NBR 13170, Rio de Janeiro, 1994.

ARAUCO, E.M.R.; FUNARI, S.R.C.; DUCATTI, C.; SILVA, E.T.; VENTURINI FILHO, W.G.; VANDERLINDE, R. Variabilidade isotópica do carbono $(\delta 13 C)$ em méis brasileiros utilizando sua proteína como padrão interno. Brazilian Journal of Food Technology, v.11, n.4, p. 299304, 2008.

BASTOS, D.H.M.; FRANCO, M.R.B.; DA SILVA, M.A.A.P.; JANZANTTI, N.S.; MARQUES, M.O.M. Composição de voláteis e perfil de aroma e sabor de méis de eucalipto e de laranja. Ciência e Tecnologia de Alimentos, Campinas, v.22, n.2, p. 122-129, 2002. 
BRASIL, Instrução normativa $n^{\circ} 11$, de 20 de outubro de 2000. Regulamento técnico de identidade e qualidade do mel. Diário Oficial [da] República Federativa do Brasil, Brasília, DF, 23 out. 2000, seção 1, p. 16-17.

CAMPOS, G.; DELLA-MODESTA, R.C.; SILVA, T.J.P.; BAPTISTA, K.E.; GOMIDES, M.F.; GODOY, R.L. Classificação do mel floral ou mel de melato. Ciência e Tecnologia de Alimentos, Campinas, v.23, n.1, p.1-5, 2003.

COMISSÃO Nacional de normas e padrões para alimentos. Resolução n¹2, de 1978. Agência Nacional de Vigilância Nacional, 1978.

GALLI, D.C.; BILHALVA, A.B.; RODRIGUES, R.S.; RODRIGUES, L.S. Influência da composição do xarope nas características físico-químicas de pêssegos tipo passa. Revista Brasileira de Agrociência, v.2, n.3, p. 179-182, 1996.

MAPA. MINISTÉRIO DA AGRICULTURA, PECUÁRIA E ABASTECIMENTO. Defesa Animal. Legislações. Legislação por Assunto. Legislação de Produtos Apícolas e Derivados. Instrução Normativa n. 11, de 20 de outubro de 2000.

MARCHINI, L.C.; MORETI, A.C.C.C.; OTSUK, I.P. Análise de agrupamento, com base na composição físico-química, de amostras de méis produzidos por Apis mellifera L. no Estado de São Paulo. Ciência e Tecnologia de Alimentos, v.25, n.1, p. 8-17, 2005.

MESQUITA, L.X.; MARACAJÁ, P.B.; SAKAMOTO, S.M.; PEREIRA, T.F.C.; PEREIRA, D.S. Análise sensorial do mel de jandaíra puro (Melipona subnitida) e com misturas. Revista Verde, v.2, n.2, p. 107-112, 2007.

MODESTA, R.C.D. A cor do mel. 2007. Disponível em <http://www.agronline.com.br/artigos/artigo.php?id=358\&pg=2\&n=2> Acesso em Março, 2012.

MORALES, V.; CORZO, N.; SANZ, M.L. HPAEC-PAD oligosaccharide analysis to detect adulterations of honey with sugar syrups. Food Chemistry, v.107, n.2 p. 922-928, 2008.

PEREIRA, F.M.; LOPES, M.T.R.; CAMARGO, R.C.R.; VILELA, S.L.O. Produção de mel. Embrapa Meio-Norte Sistemas de produção v.3, 2003. Disponível em <http://sistemasdeproducao.cnptia.embrapa.br/FontesHTML/Mel/SPMel/index.htm> Acesso em Março 2012.

PIANA, M.L.; PERSANA ODDO, L.; BENTABOL, A.; BRUNEAU, E.; BOGDANOV, S.;DECLERCK, C.G. Sensory analysis applied to honey: state of the art. Apidologie, v.35, n.1, p. 26-37, 2004.

SALGADO, T.B.; ORSI, R.O.; FUNARI, S.R.C.; MARTINS, A. Análise físico-química de méis de abelhas Apis mellifera L. comercializados na região de Botucatu, São Paulo, Brasil. PUBVET, v.2, n.20, 2008.

SEBRAE. Apicultura. Exportações em alta em 2011. Disponível em< http://www.sebrae.com.br/setor/apicultura/sobreapicultura/mercado/exportacoes/integra_bia/ ident_unico/18783 >. Acesso em Março, 2012.

SERRA, M.C.C. As propriedades antioxidantes do mel. Centro de estudos de engenharia química. Instituto Superior de Engenharia de Lisboa. Disponível em<http://www.lousamel.pt/files/noticias/pdf/38_pdf_propriedades_anti_oxidantes_do_mel.p df $>$ Acesso em Março, 2012.

VENTURINI, K.S.; SARCINELLI, M.F.; SILVA, L.C. Características do mel. Boletim Técnico PIE:UFES:01107. Pró-reitoria de extensão-Programa Institucional de extensão. Universidade Federal do Espírito Santo. 2007 
WHITE Jr., W.; DONER, L.W. Mass spectrometric detection of high-fructose corn syrup in honey by use of $13 \mathrm{C} / 12 \mathrm{C}$ ratio: collaborative study. Journal of the Association of Official Analytical Chemists, New Jersey, v. 61, p. 746-50, 1978. 